Research Article

\title{
Effective Denoising of Firing Shock Force on Shoulder by Morphological Wavelet
}

\author{
Ye Lu $\mathbb{D},{ }^{1}$ Ke-dong Zhou $\mathbb{D}^{1},{ }^{1}$ Peng-han Gong $\mathbb{D}^{2},{ }^{2}$ and Bing Li $\mathbb{D}^{2}$ \\ ${ }^{1}$ School of Mechanical Engineering, Nanjing University of Science and Technology, Nanjing 210094, Jiangsu, China \\ ${ }^{2}$ First Department, Mechanical Engineering College, No. 97, He-ping West Road, Shi Jia-zhuang 050003, He Bei Province, China
}

Correspondence should be addressed to Ke-dong Zhou; zkd81151@njust.edu.cn

Received 31 July 2018; Revised 9 November 2018; Accepted 21 November 2018; Published 13 December 2018

Academic Editor: Franck Poisson

Copyright (c) 2018 Ye Lu et al. This is an open access article distributed under the Creative Commons Attribution License, which permits unrestricted use, distribution, and reproduction in any medium, provided the original work is properly cited.

Wavelet transform is one of the most desirable tools for depressing noise. However, the traditional linear wavelets are not always suitable for any real world signals with strong background noises. In this work, we present a new morphological wavelet, named averaged dilation-erosion morphological wavelet (ADEMW), for depressing the noise in signals of firing shock force on the shoulder. Simulated signals with different SNRs are generated to evaluate and compare the proposed new wavelet scheme with the traditional linear wavelet and another two morphological wavelets presented in literature. Experimental results reveal that the presented ADEMW gives the most promising noise suppression performance. Then, the ADEMW is employed to process the realworld signals acquired from a firing shock force testing system. Processing results demonstrate that the ADEMW also outperforms another three wavelets obviously for depressing the strong background noise in the signals of firing shock force on the shoulder. The main impulsive components in the firing shock force can be clearly detected for analyzing the impacts on shoulder during the shooting process. The presented ADEMW scheme has provided a novel desirable tool for analyzing the complicated signals with strong noise.

\section{Introduction}

Firing shock force on shoulder is a human-rifle interaction force, which is generated between the buttstock and the shooter's shoulder during the shooting process of a handheld rifle. The recoil force is the source power of firing shock force on the shoulder. The precise measurement of this force during shooting is one of the most significant issues in the research of human-rifle interaction system $[1,2]$. It can provide sufficient information for the construction of a human-rifle interaction model, which will lead to improvements on the design and use of handheld weapons [3].

However, it is unavoidable to introduce noises in the measurement of firing shock force on the shoulder due to the complicated test environment and the limitation of the testing instrument. It brings serious problems for analyzing the value and trend of firing shock force on the shoulder due to the disturbance of strong noises. Therefore, how to effectively eliminate the influence of noise to the useful signal is a significant issue in this field.

Wavelet-based denoising technique is one of the most popular signal processing tools due to its flexibility and excellent capacity of depressing random noise [4-6]. Nevertheless, the traditional wavelet transforms are linear, which are based on the harmonic analysis. It is not always suitable to analyze any real-world signals, especially the complicated random noises. Thus, it is desirable to extend the traditional linear wavelets to nonlinear areas $[7,8]$.

Morphological wavelet (MW), presented by Heijmans and Goutsias [9], has provided an axiomatic framework for designing most existing linear and nonlinear wavelet decompositions. MW inherits the multidimensional and multilevel analysis of wavelet, whilst they do not require the time-frequency domain analysis. The MW extends the original wavelet from the linear domain, which is based on the convolution, to the nonlinear domain, which is based on morphological operators [10-13]. Furthermore, MW has 
incorporated the lifting scheme, which is an entirely space domain wavelet construction approach $[14,15]$. It offers the possibility of replacing linear filters by nonlinear ones, such as rank-order filters or morphological operators $[9,16]$. Therefore, it has provided a flexible tool for designing various morphological wavelet transforms.

The main advantage of MW presented before is its ability to preserve the edge or gradient in the image or signal with sudden changes. But, it is not suitable for denoising because the filters involved in the original MW are not designed for depressing the noise. Thus, a novel morphological wavelet, named averaged dilation-erosion morphological wavelet (ADEMW), is proposed for depressing noise in this work. Both simulated signals and real signals of firing shock force on the shoulder are utilized to verify the effectiveness of the presented scheme. The traditional linear wavelet and another two morphological wavelets are also employed for a comparison. The experimental results have demonstrated the promising ability of our proposed ADEMW scheme to suppress the strong noise in firing shock force on the shoulder. Therefore, we can analyze the main impact exerted on the shoulder during the shooting process more effectively.

The remainders of this work are organized as follows. Section 2 gives a brief review about MW and lifting scheme. The proposed ADEMW scheme is also described. In Section 3, simulated signals with different SNRs are constructed to evaluate the proposed technique. And, we apply the presented ADEMW scheme to the analysis of firing shock force on the shoulder in Section 4. The conclusions of this work are summarized in Section 5.

\section{Averaged Dilation-Erosion Morphological Wavelet (ADEMW)}

2.1. Morphological Wavelet. Morphological wavelet is developed as a nonlinear multiresolution signal decomposition scheme by Heijmans and Goutsias [9]. The main difference of morphological wavelet (MW) to the traditional linear wavelet (LW) is its signal analysis filter. The linear filters used in LW are replaced by morphological filters, such as erosion or dilation filters, which take the minimum or maximum as the main operators. There are some simple morphological wavelets, such as morphological Haar wavelet and morphological median wavelet, which have been developed by Heijmans. The details of these schemes can be found in [9].

Furthermore, Heijmans incorporated the lifting scheme, as shown in Figure 1, to the construction of morphological wavelets [9]. The left part is the decomposition stage, and the right part is the synthetic stage, which is the reverse of the decomposition procedure. There are two types of operators, namely, the prediction lifting and update lifting operators, in the decomposition procedure, as shown in Figure $1[14,15]$. By utilizing the signal analysis operator $\psi_{0}$ and detail analysis operator $w_{0}$, the input signal $x_{0}$ is separated to two signals, namely, the approximation signal $x_{1}$ and detail signal $y_{1}$. Then, an operator $\pi$, named prediction operator, is acting on $x_{1}$. The result is used to modify the detail signal as $y_{1}^{\prime}=y_{1} \widehat{=} \pi\left(x_{1}\right)$. Furtherly, another operator $\lambda$, named update operator, is acting on the modified detail signal $y_{1}^{\prime}$. The result is then utilized to modify the approximation signal $x_{1}$ as $x_{1}^{\prime}=x_{1}=\lambda\left(y_{1}^{\prime}\right)$. In the synthetic procedure, a reverse operation is executed and the original input signal $x_{0}$ can be reconstructed perfectly in this way.

Based on the lifting scheme, there are some interesting morphological lifting schemes, such as max-lifting, minlifting, and median-lifting schemes, have been constructed, and the detail of these schemes can also be found in [9].

2.2. Averaged Dilation-Erosion Morphological Wavelet. Although the morphological lifting schemes demonstrate various advantages in the field of signal analysis, there are still some limitations that exist in practice. The most significant advantage of the original MW is its excellent ability to process sudden changes, such as impulses and edges in the input signal. The filters designed by original MW are not specialized for suppressing noise. Therefore, based on the morphological lifting wavelets described above, an averaged dilation-erosion morphological wavelet (ADEMW) is developed in this work to depress the noise, especially the background noise introduced in the firing shock force measuring process.

The main idea of ADEMW is using the averaged morphological dilation-erosion filter as the updating operator, and there is no predicting in this scheme:

$$
\begin{aligned}
y^{\prime}(n)= & y(n), \\
x^{\prime}(n)= & x(n)+0.5 *(y(n-1) \vee x(n) \vee y(n)) \\
& +(y(n-1) \wedge x(n) \wedge y(n)) .
\end{aligned}
$$

In this way, we construct an averaged dilation-erosion morphological wavelet (ADEMW) by utilizing the lifting scheme described above. It can be observed that, the local maximum and local minimum values are averaged by the updating filter. According to the research of morphological filters, this operator can depress noise effectively. Therefore, this ADEMW scheme is capable of depressing random noise.

\section{Simulated Signal Analysis}

In this section, simulated signals with different SNRs are generated to evaluate the performance of the presented ADEMW method on depressing noises. Moreover, the traditional linear wavelet transforms and another two morphological wavelets, namely, the maximum morphological wavelet and the median morphological wavelet, are also utilized to compare with the ADEMW technique.

The sampling frequency is $4096 \mathrm{~Hz}$, and the sampling time is $1 \mathrm{~s}$. The original simulated signal with no noise is shown in Figure 2(a). Two typical contaminated signals with different SNRs, namely, $25 \mathrm{~dB}$ and $15 \mathrm{~dB}$, are displayed as Figures 2(b) and 2(c), respectively. The corresponding frequency spectrum of the three signals in Figure 2 is also given in Figure 3. It can be found that the main energy is 


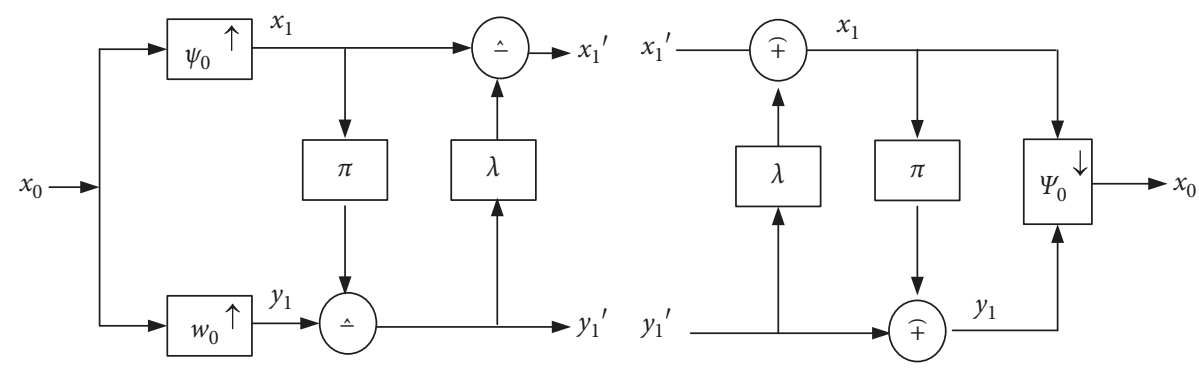

FIgURE 1: The schematic of lifting scheme.

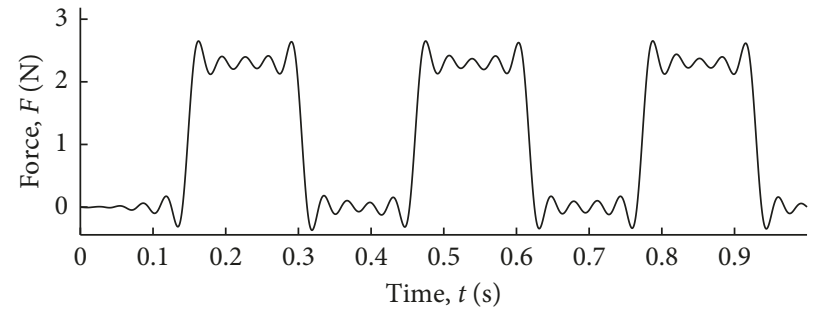

(a)

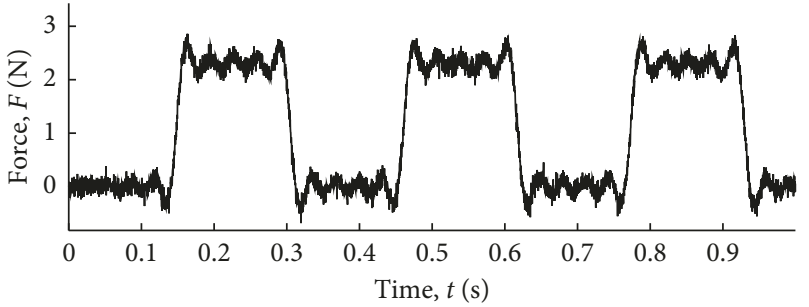

(b)

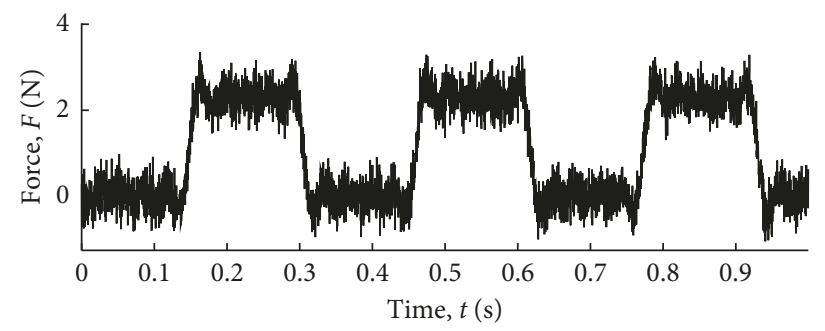

(c)

FIgURE 2: The simulated signals with different SNRs: (a) signal without noise; (b) signal added noise with SNR $25 \mathrm{~dB}$; (c) signal added noise with SNR $15 \mathrm{~dB}$.

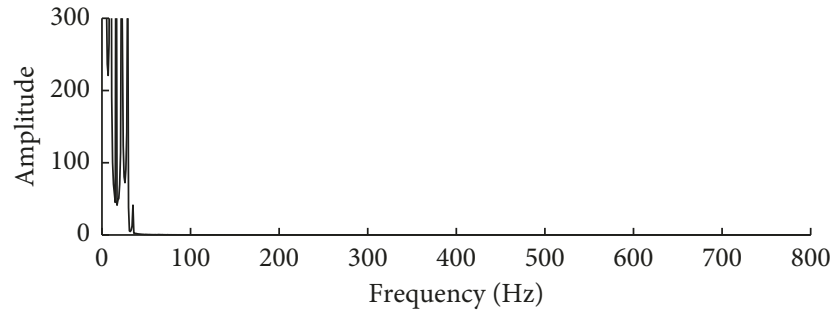

(a)

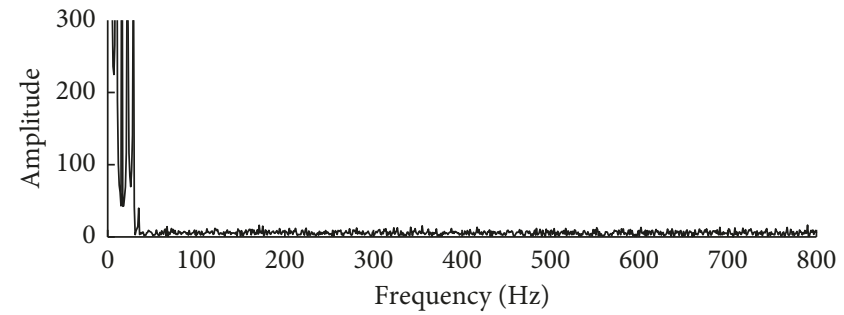

(b)

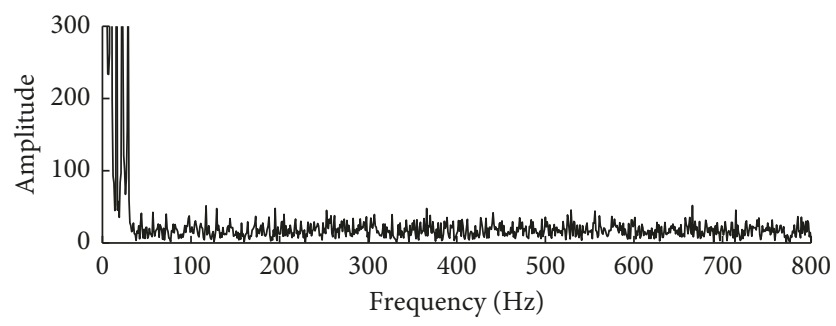

(c)

FIgURE 3: The corresponding frequency of simulated signals with different SNRs: (a) signal without noise; (b) signal added noise with SNR $25 \mathrm{~dB}$; (c) signal added noise with SNR $15 \mathrm{~dB}$. 
concentrated in the band $0-40 \mathrm{~Hz}$. There are no frequency components appearing in other bands in the frequency spectrum of original signal. But, with the contaminated signals, the "noise frequencies" appeared in all bands. The energy of the "noise frequencies" is also increasing along with the noise level. It should be mentioned that the energy in the band $0-40$ is very large, so the maximum energy displayed in Figure 3 is set to be 300 to demonstrate the "noise frequencies" more clearly. Also, the displayed frequency band is set to be $0-800 \mathrm{~Hz}$ for the same purpose.

The four denoising methods mentioned above are utilized to depress the noise. The decomposition level is set to be 3 for all the wavelets. The mother wavelet of traditional linear wavelet is set to be "db5," and the "SURE" thresholding criterion is selected. It should be noted that there are no more parameters needed to be set for all the three morphological wavelets.

The denoising results of the two typical contaminated signals in Figure 2 by utilizing the abovementioned four wavelet methods are demonstrated in Figures 4 and 5, respectively. And, the corresponding frequency spectrums are given in Figures 6 and 7, respectively.

It can be clearly observing that the proposed ADEMW yields the most favorable denoising effects among the four methods. The noises are depressed effectively, and the main components of the signal are retained. The "noise frequencies" are also depressed more effectively than other methods, as shown in Figures 6 and 7. The traditional linear wavelet also gives desirable results, but there still exist some noises in processed signals, especially when the SNR is low. The morphological median wavelet gives the worst denoising results according to our experiments.

\section{Analyzing the Signals of Firing Shock Force on Shoulder by ADEMW}

4.1. Testing System of Firing Shock Force on Shoulder. The testing system of firing shock force on the shoulder consists of three-axis force transducer, data acquisition system, and a computer. The three axis force transducer is fixed on the shooter's shoulder. Figure 8 displays the schematic of the constructed testing system of firing shock force, and Figure 9 is the transducer and its place during firing shock force testing. The transducer used in our test is the ME K3D120 three-axis force transducer and its parameters are given in Table 1.

The sample frequency was set to be $30 \mathrm{kHz}$, and the sample points were set to be 8192 points. It means that, for each signal, the acquisition time window is about $0.273 \mathrm{~s}$. Figure 10 demonstrates the waveforms of the signals on three directions, namely, the $x$-axis, $y$-axis, and $z$-axis, which measure the forces produced in direction of front-back, leftright, and up-down, respectively. It can be found that the signals are seriously disturbed by the noise generated in the testing process. It is very desirable to eliminate the influence of noise to the precise analysis of firing shock force on the shoulder.
4.2. Noise Depression for Signals of Firing Shock Force on Shoulder. Therefore, the presented ADEMW is employed to depress the noise in the signals of firing shock force on the shoulder. Also, as described in Section 3, the traditional linear wavelet and another two morphological wavelets, namely, the maximum morphological wavelet and the median morphological wavelet, are employed and compared with the proposed ADEMW. The parameters of all wavelet methods are set to be same with the parameters in Section 3.

Figures 11-13 demonstrate the denoising results of signals along three axes by using the four methods described above. It can be clearly found that the proposed ADEMW gives the best results in all the three signals. The noises are depressed effectively and the impulsive components can be clearly demonstrated in the processed results.

4.3. Analysis of the Firing Shock Force on Shoulder. As shown in Figures 11-13, the shoulder force along the $x$-axis, that is, the direction of front-back, is the main force generated during the shooting. The shoulder forces along another two directions are obviously softer and not varied dramatically. This phenomenon is highly consistent with the actual situation in our tests.

Figure 14 displays the local signal of the shoulder force along the $x$-axis in one shoot cycle. At first, the force is about $150 \mathrm{~N}$, which is the preloaded shoulder force before shooting. Then, a shark pulse, shown as mark 1 , is emerging. This impulse is generated by the shock of firearm due to the exploding of gunpowder. After that, when the bullet passes through the gas port, the impact of propellant firing gas to the front of the gas chamber will propel the firearm forward. Therefore, the shock force declines rapidly, shown as mark 2 in Figure 14. Then, the automata move backward and knock the back end of the cartridge receiver. Thus, the shock force is increasing speedy to produce another peak, shown as mark 3. At last, the automata move forward and impact the forepart of the cartridge receiver. Due to this impact, the shock force decreases, shown as mark 4 in Figure 14.

\section{Conclusion}

This investigation has proposed a novel noise-depressing scheme, named averaged dilation-erosion morphological wavelet (ADEMW), for analyzing firing shock forces on shoulder.

The simulated signals disturbed by different degree noises are firstly employed to evaluate the proposed scheme. The traditional linear wavelet (LW) and another two morphological wavelets (MWs), named maximum morphological wavelet and median morphological wavelet, developed in literature are also used for comparison. Results have revealed that the developed ADEMW outperforms the traditional LW and MWs obviously on depressing noise.

Then, the real signals of firing shock force on the shoulder with strong background noise acquired from a testing system are processed by the presented ADEMW scheme as well as the LW and MWs mentioned above. 


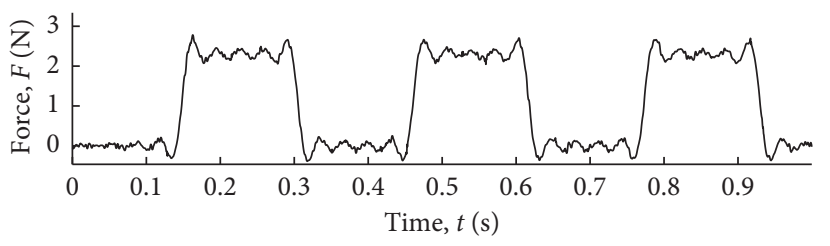

(a)

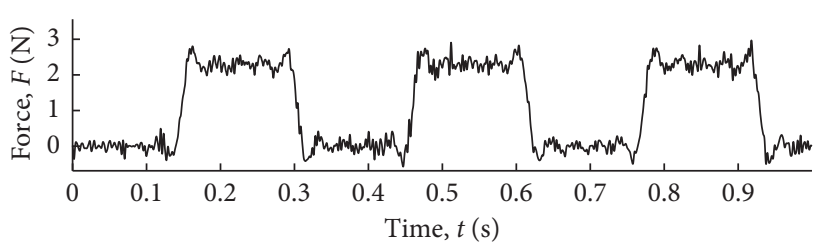

(c)

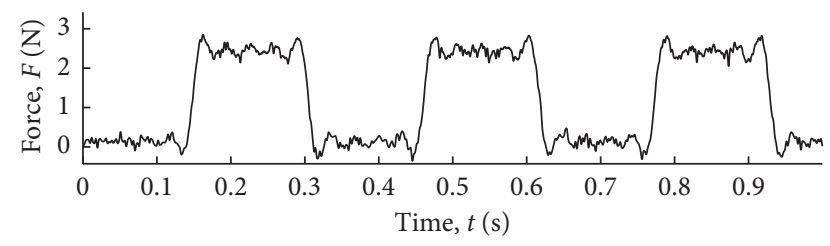

(b)

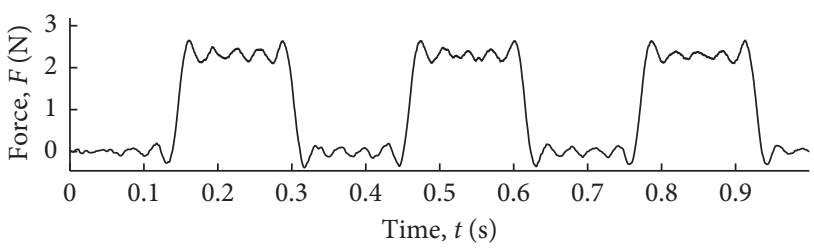

(d)

FIgURE 4: The denoising results on signal with SNR $25 \mathrm{~dB}$ by using (a) linear wavelet, (b) maximum morphological wavelet, (c) median morphological wavelet, and (d) presented ADEMW.

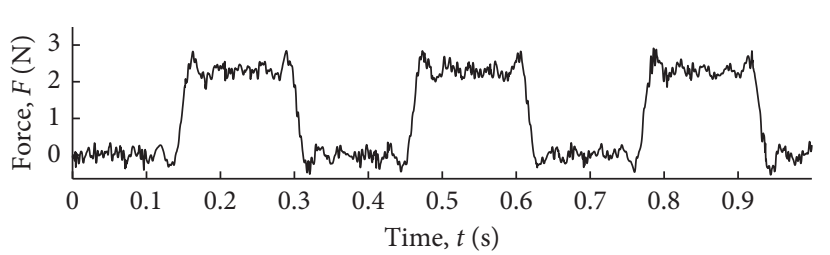

(a)

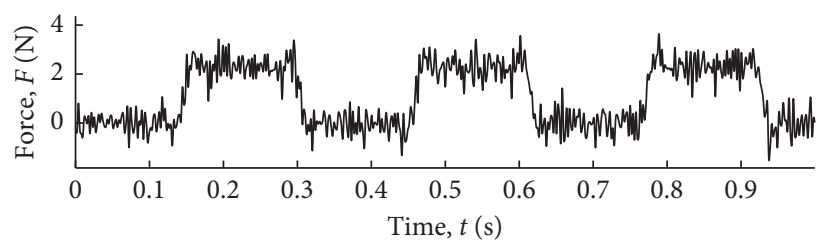

(c)

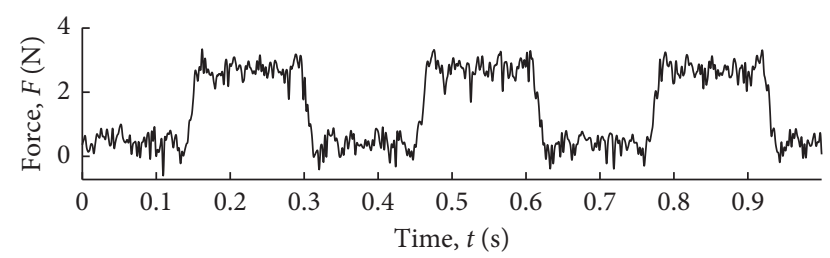

(b)

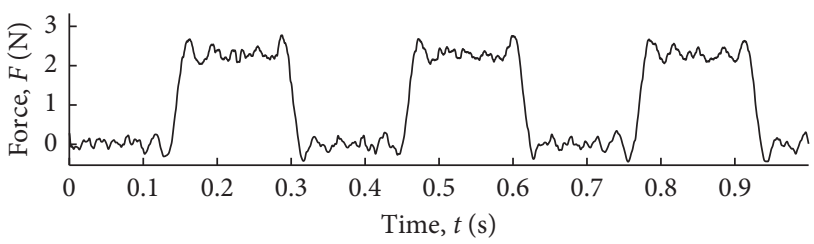

(d)

FIgURE 5: The denoising results on signal with SNR $15 \mathrm{~dB}$ by using (a) linear wavelet, (b) maximum morphological wavelet, (c) median morphological wavelet, and (d) presented ADEMW.

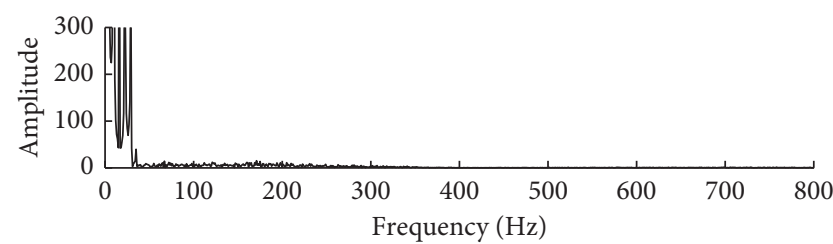

(a)

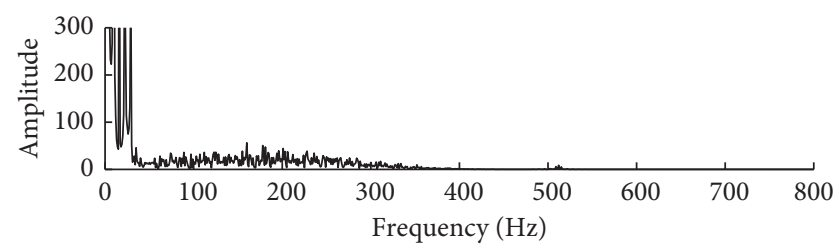

(c)

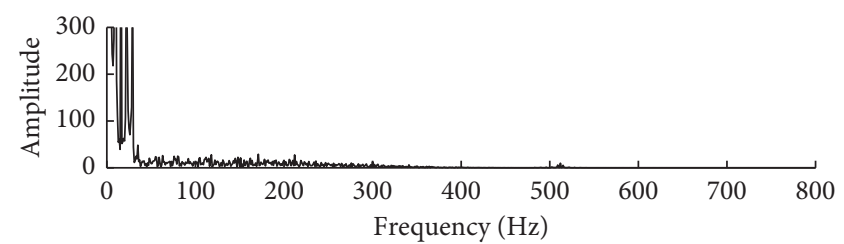

(b)

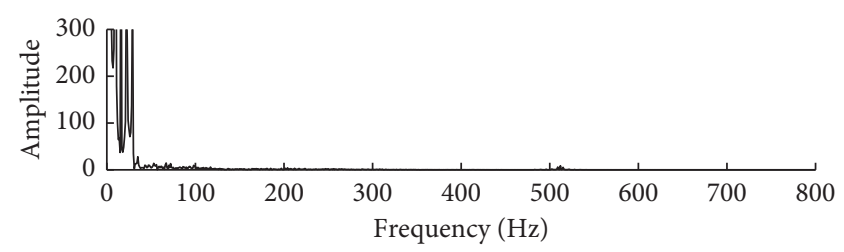

(d)

FIGURE 6: The corresponding frequency of denoised signal with SNR $25 \mathrm{~dB}$ by using (a) linear wavelet, (b) maximum morphological wavelet, (c) median morphological wavelet, and (d) presented ADEMW. 


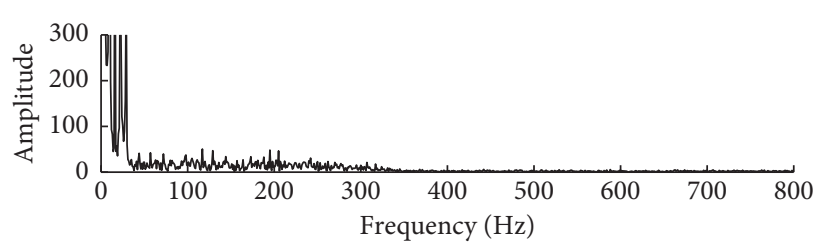

(a)

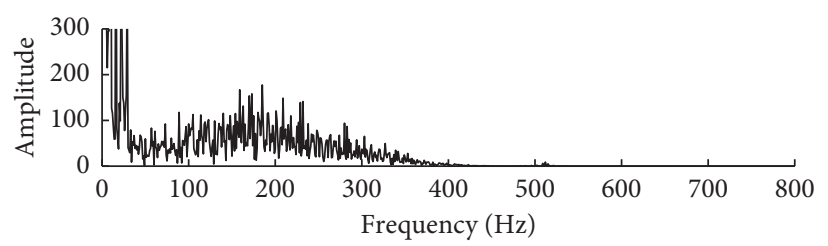

(c)

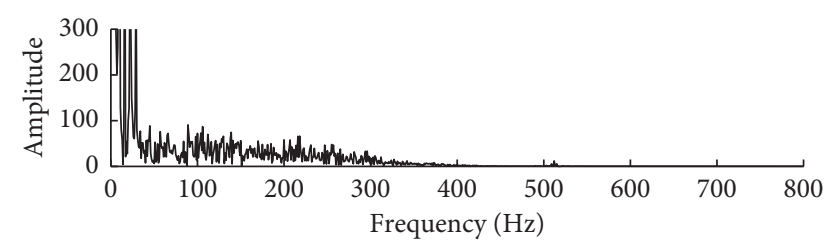

(b)

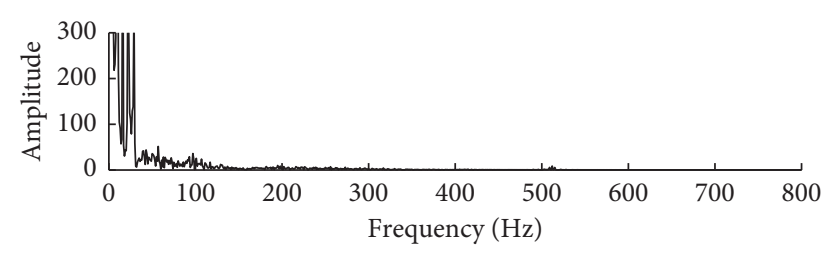

(d)

FIGURE 7: The corresponding frequency of denoised signal with SNR $15 \mathrm{~dB}$ by using (a) linear wavelet, (b) maximum morphological wavelet, (c) median morphological wavelet, and (d) presented ADEMW.

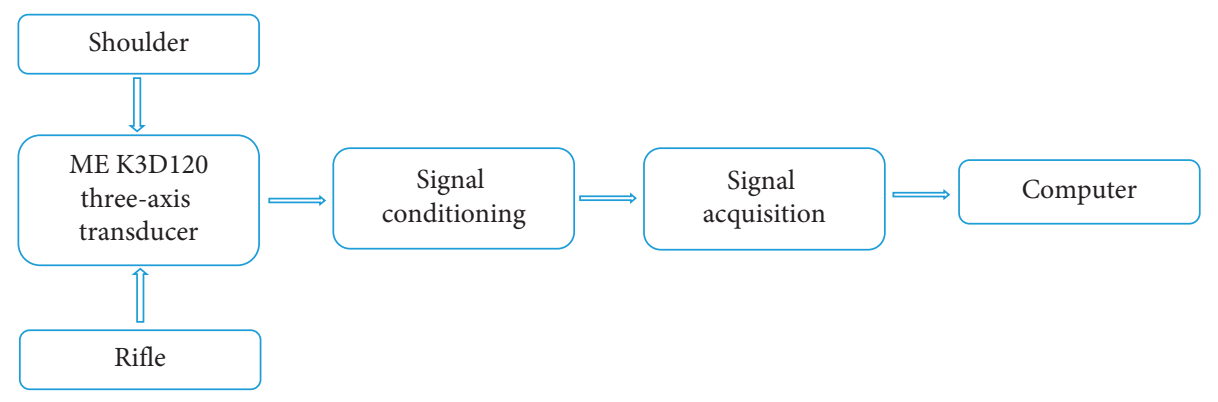

FIGURE 8: The schematic of testing system of firing shock force on shoulder.
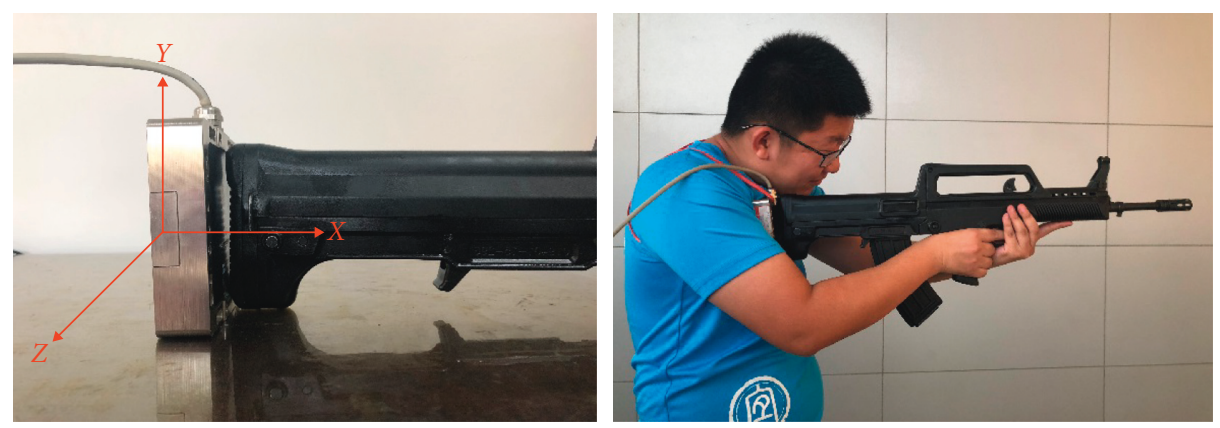

FIGURE 9: The transducer and its place in the testing.

TABle 1: Parameters of the transducer used in the test

\begin{tabular}{lccccc}
\hline Type & Range $(N)$ & Accuracy & Linear error & Size $(\mathrm{mm})$ & Working temperature $\left({ }^{\circ} \mathrm{C}\right)$ \\
\hline ME K3D120 & \pm 1000 & $0.5 \%$ & $0.2 \%$ & $120 * 120 * 30$ & $-20 \sim 60$ \\
\hline
\end{tabular}

Experimental results have demonstrated that the proposed ADEMW scheme achieves the most promising performance for depressing the strong background noise.

The main reason to the results obtained above lies in that the updating operator used in ADEMW is a new filter designed for depressing noise. This new filter is obtained by computing the average of local minimum and maximum of the analyzed signal. The traditional MW, such as maximum morphological wavelet and median morphological wavelet, which using maximum filter or median filter as updating operators, is more suitable for maintaining original signal structures than depressing the noise.

According to the results obtained above, it can be concluded that the presented ADEMW scheme demonstrates to be an attractive approach for depressing firing shock force signals with strong background noise. 


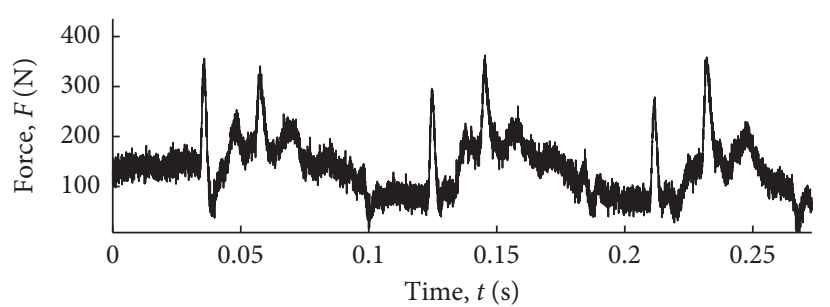

(a)

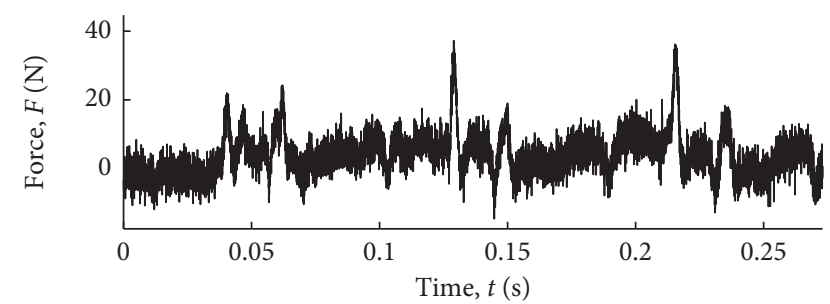

(b)

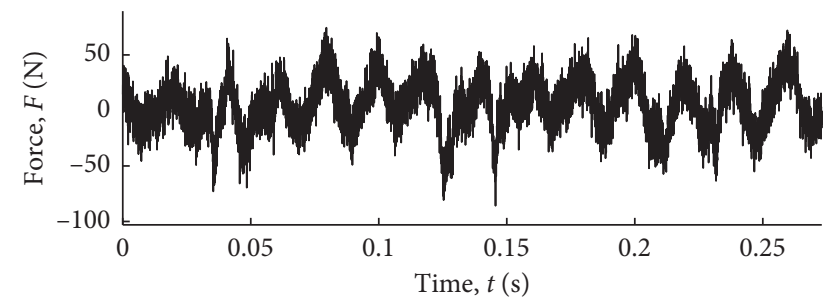

(c)

FIGURE 10: Original signals of firing shock force on shoulder in three directions: (a) $x$-axis (front-back); (b) $y$-axis (up-down); (c) $z$-axis (leftright).

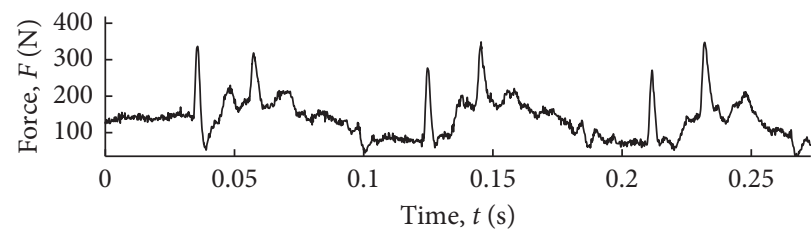

(a)

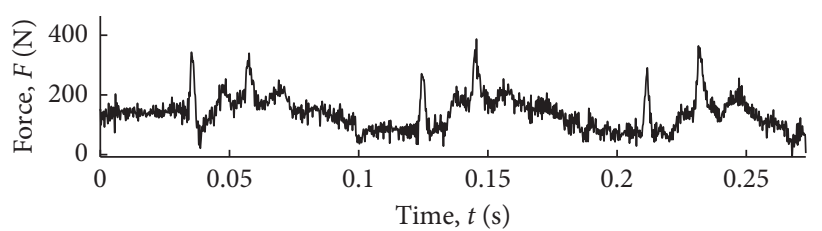

(c)

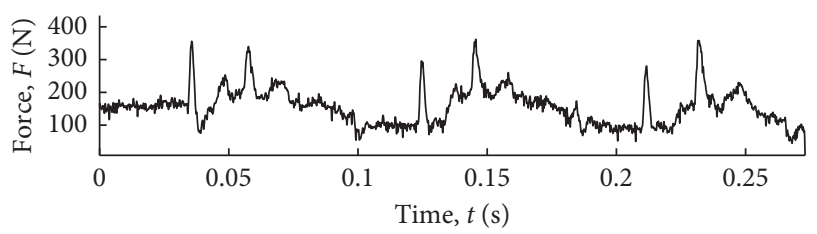

(b)

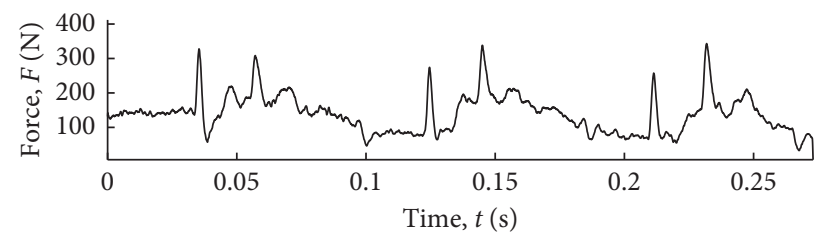

(d)

Figure 11: The denoising results on signal of firing shock force on shoulder along $x$-axis: (a) linear wavelet; (b) maximum morphological wavelet; (c) median morphological wavelet; (d) presented ADEMW.

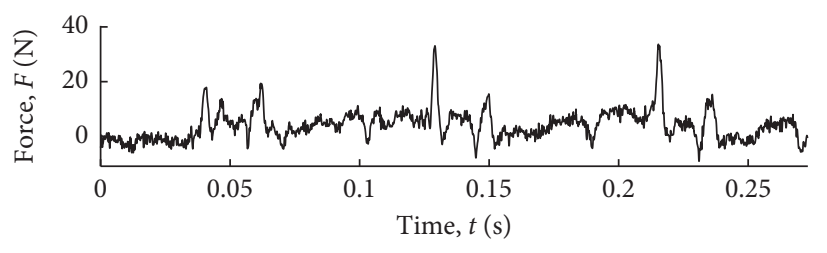

(a)

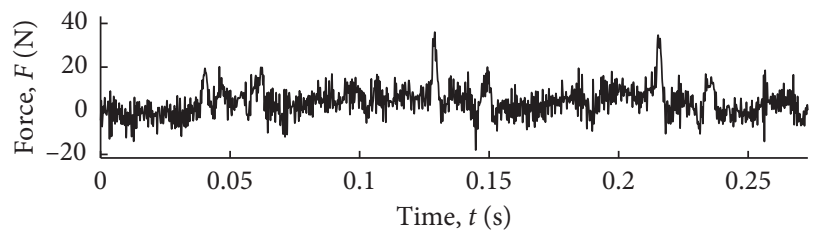

(c)

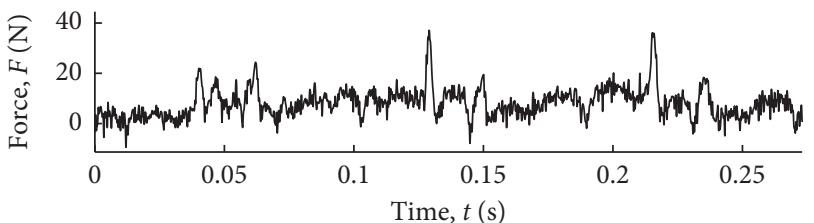

(b)

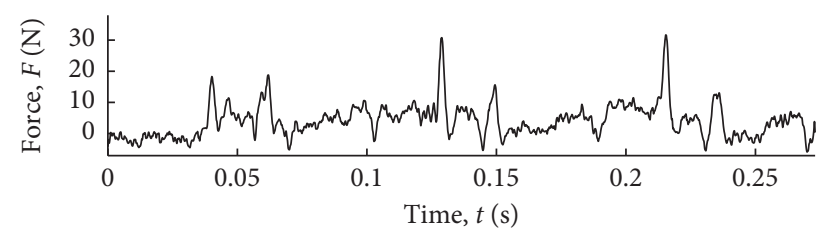

(d)

FIGURE 12: The denoising results on signal of firing shock force on shoulder along $y$-axis: (a) linear wavelet; (b) maximum morphological wavelet; (c) median morphological wavelet; (d) presented ADEMW. 


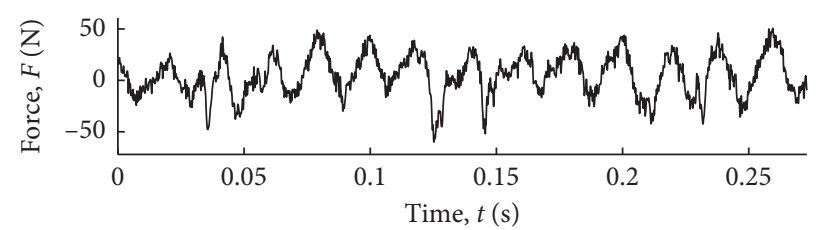

(a)

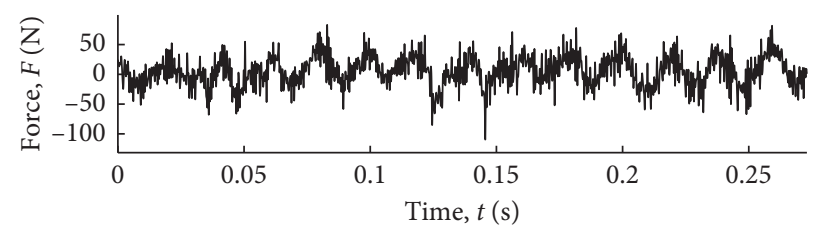

(c)

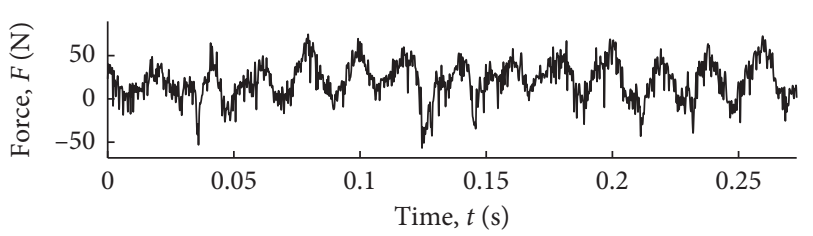

(b)

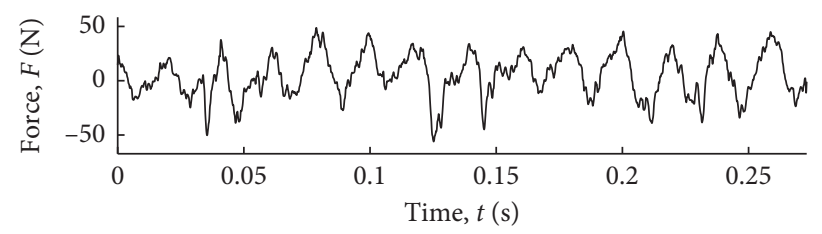

(d)

FIgURE 13: The denoising results on signal of firing shock force on shoulder along $z$-axis: (a) linear wavelet; (b) maximum morphological wavelet; (c) median morphological wavelet; (d) presented ADEMW.

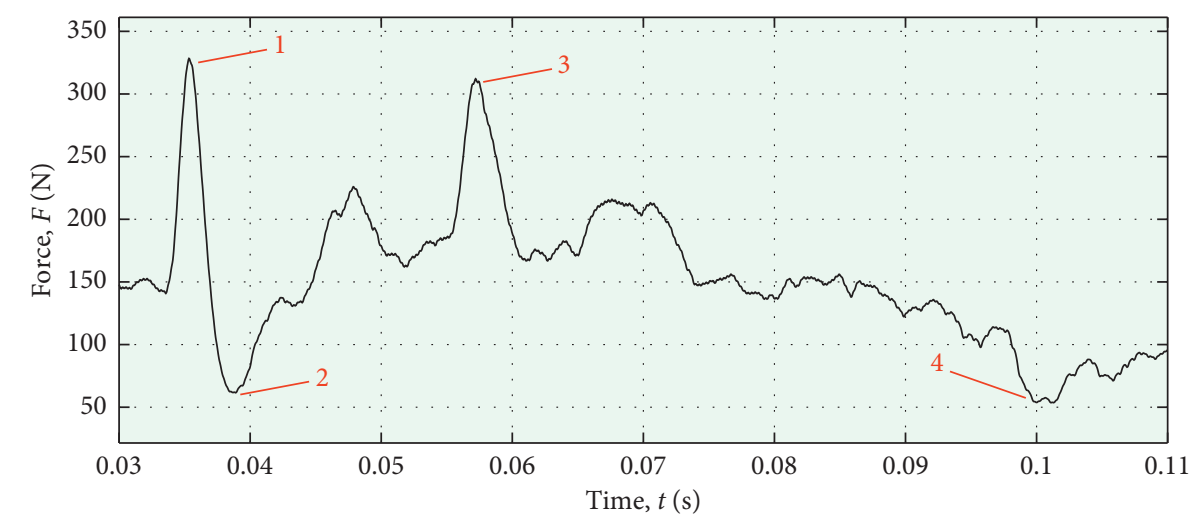

FIGURE 14: The local signal of firing shock force on shoulder along $x$-axis in one shoot cycle.

Consequently, the main impact exerted on the shoulder during the shooting process can be analyzed more effectively. Otherwise, the presented ADEMW can be easily extended to analyze other real-world signals with noises.

\section{Data Availability}

The data used to support the findings of this study are available from the corresponding author upon request.

\section{Conflicts of Interest}

The authors declare that they have no conflicts of interest.

\section{Acknowledgments}

This research was supported by the Chinese Postdoctoral Science Foundation (No. 2018T110503) and National Natural Science Foundation of China (No. 51205405).

\section{References}

[1] M. J. Hall, "Measuring felt recoil of sporting arms," International Journal of Impact Engineering, vol. 35, no. 6, pp. 540-548, 2008.
[2] B. Lonzi, M. Martarelli, C. Santolini, and L. Scalise, "Measurement of firing impulse force in rifles," in Proceedings of International Conference on Noise And Vibration Engineering, P. Sas, D. Moens, and H. Denayer, Eds., pp. 2065-2075, Katholieke University Leuven, Department Werktuigkunde, Leuven, Belgium, September 2014.

[3] F. Morelli, J. M. Neugebauer, M. E. LaFiandra, P. Burcham, and C. T. Gordon, "Recoil measurement, mitigation techniques, and effects on small arms weapon design and marksmanship performance," IEEE Transactions on HumanMachine Systems, vol. 44, no. 3, pp. 422-428, 2014.

[4] Y. L. Chen, P. L. Zhang, B. Li, and D. H. Wu, "Denoising of mechanical vibration signals using quantum-inspired adaptive wavelet shrinkage," Shock and Vibration, vol. 2014, Article ID 848097, 7 pages, 2014.

[5] M. Z. Li, Z. Q. Wang, J. Luo, Y. S. Liu, and S. Cai, "Wavelet denoising of vehicle platform vibration signal based on threshold neural network," Shock and Vibration, vol. 2017, Article ID 7962828, 12 pages, 2017.

[6] Y. Wang, S. J. Chen, L. Ge, H. X. Hu, Y. Wang, and L. Zhou, "The optimal wavelet threshold de-nosing method for acoustic emission signals during the medium strain rate damage process of concrete," Nondestructive Testing and Evaluation, vol. 32, no. 4, pp. 400-417, 2017.

[7] R. L. Claypoole, G. M. Davis, W. Sweldens, and R. G. Baraniuk, "Nonlinear wavelet transforms, for image 
coding via lifting," IEEE Transactions on Image Processing, vol. 12, no. 12, pp. 1449-1459, 2003.

[8] B. Li, P.-l. Zhang, S.-s. Mi, R.-x. Hu, and D.-s. Liu, "An adaptive morphological gradient lifting wavelet for detecting bearing defects," Mechanical Systems and Signal Processing, vol. 29, pp. 415-427, 2012.

[9] H. J. A. M. Heijmans and J. Goutsias, "Nonlinear multiresolution signal decomposition schemes-part II: morphological wavelets," IEEE Transactions on Image Processing, vol. 9, no. 11, pp. 1897-1913, 2000.

[10] J. Sun, H. R. Li, and B. H. Xu, "The morphological undecimated wavelet decomposition - Discrete cosine transform composite spectrum fusion algorithm and its application on hydraulic pumps," Measurement, vol. 94, pp. 794-805, 2016.

[11] Y. Zhang, T. Y. Ji, M. S. Li, and Q. H. Wu, "Identification of power disturbances using generalized morphological openclosing and close-opening undecimated wavelet," IEEE Transactions on Industrial Electronics, vol. 63, no. 4, pp. 2330-2339, 2016.

[12] M. H. Khakipour, A. A. Safavi, and P. Setoodeh, "Bearing fault diagnosis with morphological gradient wavelet," Journal of the Franklin Institute, vol. 354, no. 6, pp. 2465-2476, 2017.

[13] T. N. Cruz, T. M. Cruz, and W. P. Santos, "Detection and classification of lesions in mammographies using neural networks and morphological wavelets," IEEE Latin America Transactions, vol. 16, no. 3, pp. 926-932, 2018.

[14] W. Sweldens, "The lifting scheme: a custom-design construction of biorthogonal wavelets," Applied and Computational Harmonic Analysis, vol. 3, no. 2, pp. 186-200, 1996.

[15] W. Sweldens, "The lifting scheme: a construction of second generation wavelets," SIAM Journal on Mathematical Analysis, vol. 29, no. 2, pp. 511-546, 1998.

[16] G. Piella and H. J. A. M. Heijmans, "Adaptive lifting schemes with perfect reconstruction," IEEE Transactions on Signal Processing, vol. 50, no. 7, pp. 1620-1630, 2002. 


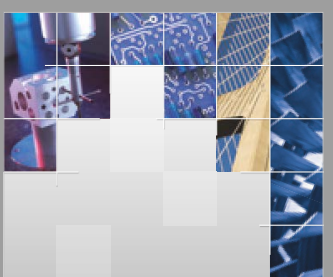

\section{Enfincering}
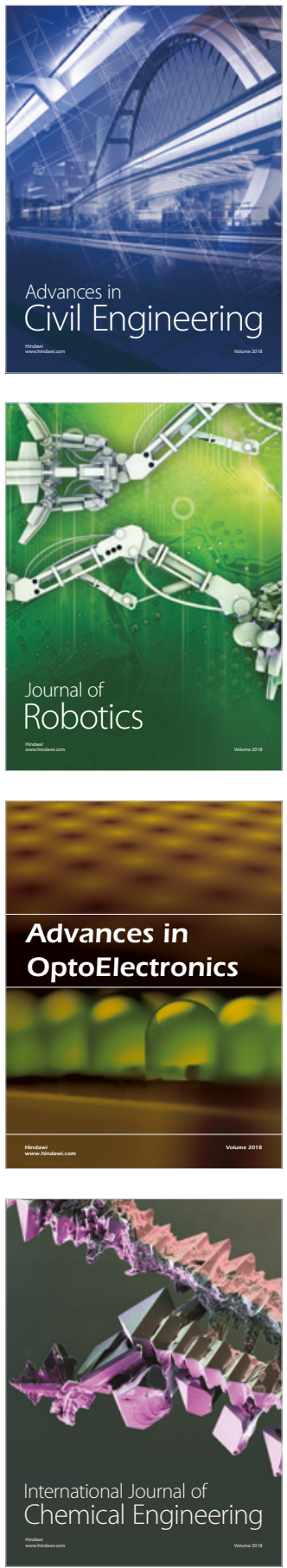

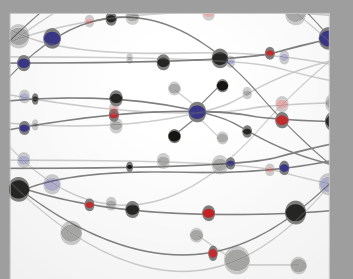

\section{Rotating \\ Machinery}

The Scientific World Journal

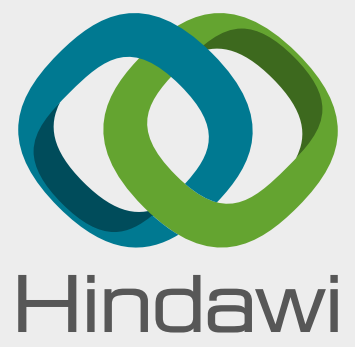

Submit your manuscripts at

www.hindawi.com
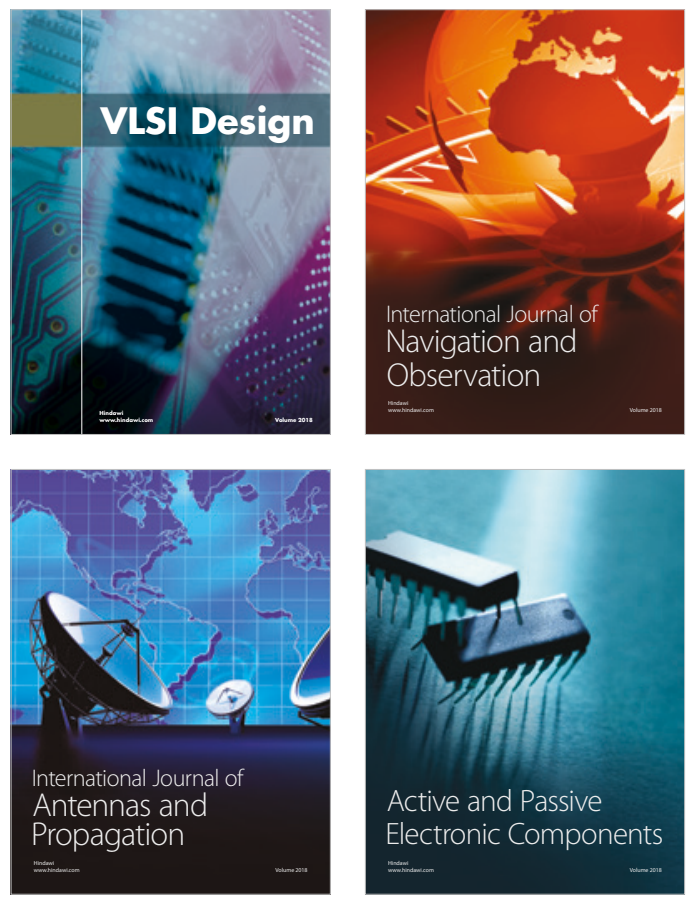
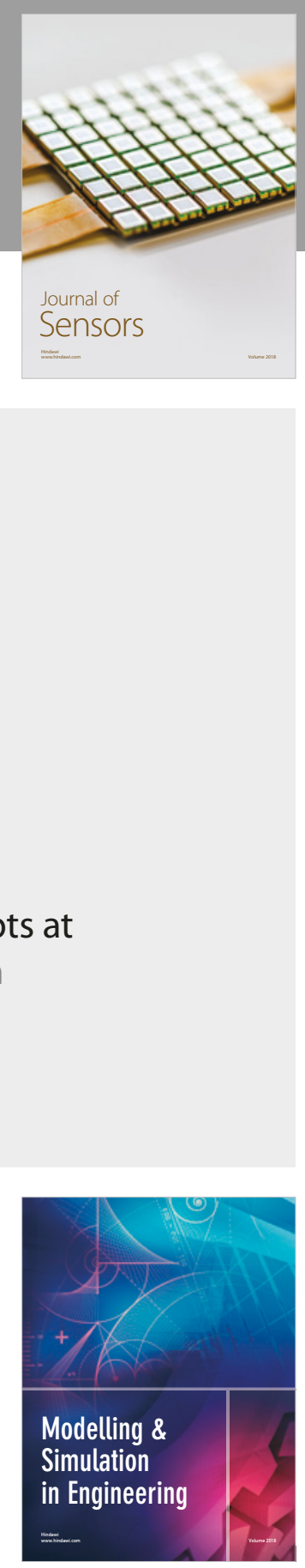

\section{Advances \\ Multimedia}
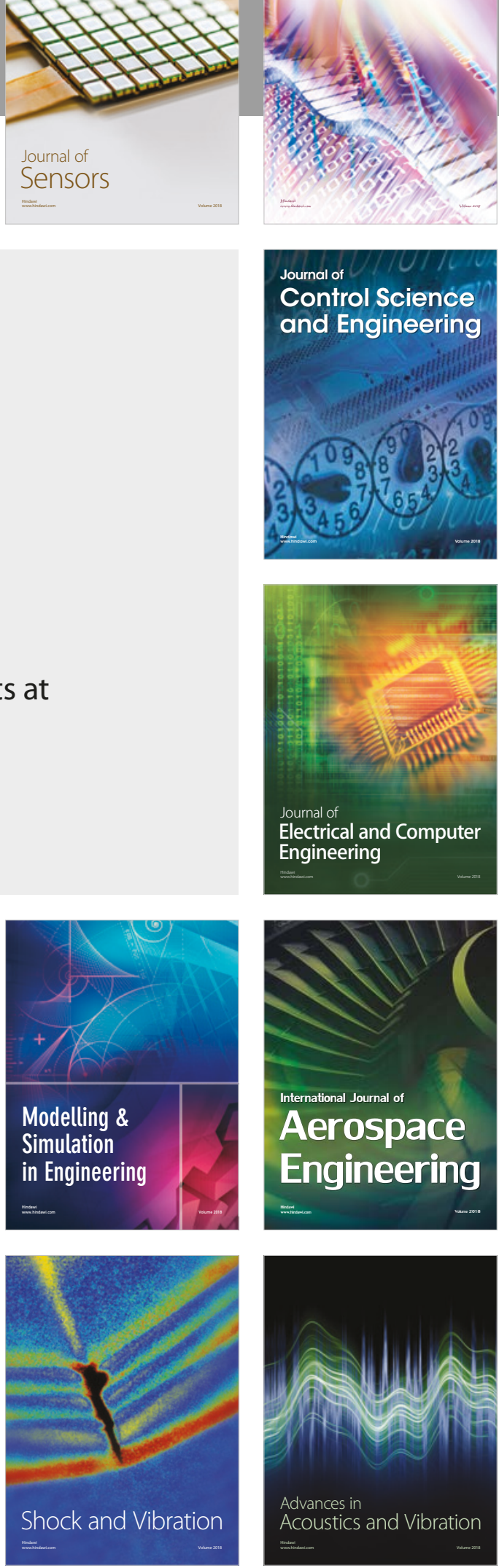